\title{
3 Research Square

\section{Principal Component Analysis of Adipocytokines and Insulin Associate with Risk Factors of Cardiovascular Diseases}

Habib yarizadeh ( $\square$ habib.yari72@yahoo.com )

https://orcid.org/0000-0003-2640-863X

Leila Setayesh

Tehran University of Medical Sciences

Moein Askarpour

Tehran University of Medical Sciences

Sara Pooyan

TUMS

Seyedeh Forough Sajjadi

tums

Negin Badrooj

TUMS

Caroline Roberts

King's College London School of Medical Education

Khadijeh Mirzaei

Tehran University of Medical Sciences

\section{Research note}

Keywords: Adipocytokines, Cardiovascular Diseases, Obesity

Posted Date: February 18th, 2020

DOI: https://doi.org/10.21203/rs.2.18283/v2

License: (c) (1) This work is licensed under a Creative Commons Attribution 4.0 International License.

Read Full License

Version of Record: A version of this preprint was published at BMC Research Notes on April 14th, 2020. See the published version at https://doi.org/10.1186/s13104-020-04976-9. 


\section{Abstract}

Objectives : Obesity plays an important role in the development of chronic diseases like cardiovascular diseases and diabetes. The possible underlying mechanism for this connection is that adipose tissue secretes an array of chemical messenger adipokines proinflammatory cytokines (tumor necrosis factoralpha, interleukin-6, and interleukin-1-beta). This study aimed to investigate the linkage between adipocytokines and insulin with the cardiovascular disease risk, with particular reference to the adipokines galectin-3, plasminogen activator inhibitor-1, interleukin-1-beta, C-reactive protein, and monocyte chemoattractant protein. Result: Two patterns were identified. The first pattern was galectin-3, plasminogen activator inhibitor-1 and interleukin-1-beta and the second one was C-reactive protein, insulin and monocyte chemoattractant protein-1. The second pattern was strongly associated with the higher scores for resting metabolic rate, diastolic blood pressure, homeostasis model insulin resistance index, lipid profile (except low density lipoprotein, total cholesterol), and body composition parameters (except fat free mass index and waist hip ratio), while negatively associated with age and high density lipoprotein level (all $p<0.05)$. The first pattern was, however, significantly associated with body fat mass, obesity degree percentage, waist circumference, fat mass index, and waist hip ratio $(P<0.05$ for all).

\section{Introduction}

Cardiovascular diseases (CVDs) is an umbrella term that includes diseases affecting the heart and blood vessels [1]. There are a number of modifiable (smoking, alcohol consumption, diet, exercise) and nonmodifiable (genes) that may contribute to the onset and progression of CVD [2], with strong evidence suggesting obesity is a major risk factor for CVD. Relevant reports also demonstrated that obesity is predominant in CVD patients [3]. one possible underlying mechanism for this connection is that adipose tissue secretes an array of chemical messenger adipokines including leptin and proinflammatory cytokines (tumor necrosis factor-alpha (TNFa), interleukin-6 (IL-6), interleukin-1- beta (IL-1 $\beta$ )) [4]. Indeed, abnormal levels of adipokines may predispose obese individuals to cardiovascular diseases [5].

A high proportion of body fat mass, in particular abdominal fat is one of the major risk factors for CVD [12], and as a result, obesity is an independent underlying predictor of CVD which is often related to elevated levels of inflammatory mediators [6, 7]. These inflammatory mediators are regarded as independent risk factors for future coronary events [7]. Proinflammatory cytokines are capable of triggering the synthesis of C-reactive protein (CRP) by hepatocytes [8]. There is substantial evidence supporting the role of CRP in the pathogenesis and worsening of vascular inflammation, vessel damage and clinical CVD complications [9].

Obesity has become a burgeoning epidemic in the public health [10] and is inevitably accompanied by the increased level of plasma cholesterol, triglyceride, and blood sugar as well as levels of insulin, which can elevate susceptibility to the cardiovascular diseases [11]. The aim of this study was to determine if these factors contribute to cardiovascular disease in women. 
This study aimed to investigate the linkage between serum markers of obesity and risk of cardiovascular diseases, with particular reference to the adipokines galectin-3, plasminogen activator inhibitor-1 (PAl-1), IL-1 $\beta$, CRP, monocyte chemoattractant protein-1 (MCP-1), and insulin.

\section{Methods}

\section{Subjects}

Three hundred and sixty women with body mass index (BMI) $\geq 25$ were recruited from health centers of Tehran. Investigating obese individuals aged 18 to 50. Participants were excluded if they were taking medications or had history of type one diabetes mellitus. Smoking, alcohol consumption, pregnancy, lactation, and exercise were considered as additional exclusion criteria. All participants signed a written informed consent and the study was approved by Tehran University of Medical Sciences ethics committee (IR.TUMS.VCR.REC.1395.1597).

After an overnight (12 hours) fast, volunteers attended to the clinical health centers and standard anthropometric parameters were recorded. Three readings were taken to calculate resting blood pressures at left brachial artery by an automated sphygmomanometer (Dinamap).

\section{Procedures}

Indirect calorimetry (spirometer METALYZERR 3B-R3, Cortex Biophysik GmbH, Leipzig, Germany) was used to calculate resting metabolic rate (RMR). To do so, the amount of consumed 02 and produced $\mathrm{CO} 2$ was measured after/when subjects had rested for at least 20 minutes. Gas ventilation was calibrated prior to each test.

\section{Laboratory methods}

A blood sample was also taken to assess serum concentrations of glucose (FBS), lipids, and adipokines (CRP, IL-1 $\beta$ ) using enzyme-linked immunosorbent assay kits (R\&D Systems, Techne Corporation, Minneapolis, MN) according to the manufacturer's instructions. All samples were analyzed in duplicates. The homeostasis model insulin resistance index (HOMA) was computed as the product of fasting glucose and insulin level divided by 22.5 with molar unit ( $\mathrm{mmol} / \mathrm{L})$.

\section{Statistical analysis}

All statistical analysis was conducted by the SPSS version 22 (SPSS Inc., Chicago, IL). Data are presented as arithmetic means plus their 95\% confidence interval (CI) and standard deviations unless otherwise stated.

Factor (principal component) analysis was performed to determine which plasma concentration of adipokines associated with most of observed variations. Two patterns were identified as shown in Table S1. The significance of differences between low and high value of each pattern and CVD risk factors were 
computed by analysis of variance (ANOVA) (Table S2). In addition, to keep uncorrelated factors and greater interpretability, the patterns were rotated and normalized using Varimax transformation method and Kaiser Method, respectively. Pearson's correlation coefficient indicates the association between each adipokine pattern score and CVD risk factors (Table S3). Multiple linear regression analysis was followed to calculate the incidence and strength of associations (Table 2 and $\mathbf{3}$ ). Two-sided $P$ values $<0.05$ were considered as statistically significant. Confounders including age, physical activity, energy intake, and BMI were used to adjust for linear regression analysis.

\section{Results}

\section{Subject characteristics}

Table 1 depicts main clinical and biochemical characteristics of the subjects. Mean age ranged from 18 to 50 years and BMI ranged between 25 and $40.70 \mathrm{~kg} / \mathrm{m} 2$.

\section{Correlation analysis}

Single correlation of each adipokines with clinical and biochemical risk factors of CVD are detailed in Table S3. The adipokines galectin-3 appeared related to BMI $(r=-0.109, p=0.038)$, diastolic blood pressure (DBP) $(r=-0.123, p=0.023)$, body fat mass (BFM) $(r=-0.174, p=0.003), \operatorname{ODP}(r=-0.119, p=$ 0.044), waist circumference (WC) $(r=-0.140, p=0.017)$, FMI $(r=-0.177, p=0.003)$, as well as waist-hip ratio (WHR) $(r=-.140, p=0.017)$. Similar results were obtained between PAl-1 and BMI $(r=-0.111, p=$ 0.036), DBP $(r=-0.141, p=0.009), \operatorname{BFM}(r=-0.177, p=0.003)$, ODP $(r=-0.120, p=0.041)$, WHR $(r=$ $-0.155, p=0.008)$, FMI $(r=-0.176, p=0.003)$, plus WHR $(r=-0.166, p=0.005)$. In case of CRP, the significant correlations were observed with BMI $(r=0.121, p=0.022)$, DBP $(r=0.129, p=0.018), \mathrm{HDL}(r=$ $-.152, p=0.007)$, hs.CRP $(r=0.858, p=0.0001), \operatorname{HOMA}(r=0.167, p=0.004)$, and ODP $(r=-0.133, p=$ 0.024). Neither IL-1b nor MCP-1 were significantly associated with any of the anthropocentric data, blood and body composition parameters except for a positive association between IL-1b and age $(r=0.128, p=$ $0.015)$ and negative correlation between MCP-1 and FBS $(r=-0.147, p=0.009)$.

On the other hand, insulin shown very broad correlations with age $(r=-0.146, p=0.006)$, weight $(r=0.240$, $p=0.0001), \operatorname{BMI}(r=0.232, p=0.0001), \operatorname{RMR}(r=0.317, p=0.0001), \operatorname{SBP}(r=0.112, p=0.039), \operatorname{DBP}(r=$ $0.217, p=0.0001), T G(r=0.290, p=0.0001), \operatorname{HDL}(r=-0.145, p=0.01)$, hs.CRP $(r=0.178, p=0.001)$, HOMA $(r=0.880, p=0.0001)$, FBS $(r=0.176, p=0.002)$, BFM $(r=0.227, p=0.0001)$, fat-free mass (FFM) $(r=0.292, p=0.0001)$, soft lean mass (SLM) $(r=0.278, p=0.0001)$, SMM $(r=0.307, p=0.0001)$, ODP $(r$ $=0.253, p=0.0001)$, WC $(r=0.307, p=0.0001), F M I(r=0.175, p=0.003)$, WHR $(r=0.240, p=0.0001)$.

Two patterns were identified. The first pattern was characterized by high level of galectin-3 and, PAl-1 and low level of IL-1 $\beta$. The second one was associated with CRP, insulin and MCP-1 (Table S1). As shown in Table 3, association analysis of adipokines patterns demonstrated that pattern 2 were strongly correlated with the higher scores for RMR, DBP, HOMA, lipid profile, and body composition parameters, while 
negatively associated with age and HDL level (all $\mathrm{p}<0.05$ ). However, SBP, cholesterol, LDL, FBS, FFMI, and WHR appeared unrelated to PCA2 (all $p>0.05$ ).

In contrast, neither biochemical parameters nor blood pressure of participants was significantly correlated to PCA1 pattern (all $p>0.05$ ). The first pattern was, however, significantly associated with BFM, ODP, WC, FMI, and WHR $(P<0.05$ for all).

\section{Discussion}

This study aimed to investigate the association between adipocytokines and insulin patterns with the risk of cardiovascular diseases. In this study we identified two separate adipokines patterns, explaining $56 \%$ of the total variance in the data. Of these, pattern 1 characterized by the high level of galectin-3, and PAl- 1 and low concentration of IL-1 $\beta$. The second one included CRP, MCP-1, plus insulin. In the linear regression model derived from our data, the link between the second pattern and body composition parameters, and biochemical markers of CVD was yielded significant p-values except for SBP, LDL, and FBS.

Previous investigations on the link of adipokines and CVD risk factors have been mostly limited to single adipokines $[13,14]$. But in physiology, a complex network of chemical messengers contributes to the CVD [15]. Thus, identification and evaluation of these factors in combination with each other could, at least partly, open a clear window toward prediction of the CVD. To do so, PCA was employed as a powerful statistical tool to recognize potential contributors to the observed risk factors [16]. Next, univariate regression performed to provide detailed information about quantitative associations between adipokines level and biological outcomes of the interest.

Considering the number of independent variables (adipokines plus insulin) included in this study, PCA was preferentially used for data reduction by taking a linear combination of the existing 6 orthogonal variables, allowing us to identify potentially significant patterns of adipokines plus insulin associated with CVD risk factors [17].

In consensus with the literature, our results showed that CRP, MCP-1, and insulin may be the key determinants of CVD predisposition, suggesting inflammation plays a key role in CVD development. Unraveling the molecular mechanism behind these associations may elucidate their potential for prediction of the cardiovascular events. In this respect, obesity has already been associated with lowgrade inflammation in humans $[18,19]$. The exact trigger is unknown yet [20], however, a number of systemic chemical messengers particularly resistin are released into the bloodstream [21]. Resistin (also called adipose tissue-specific secretory factor) is a hormone derived from adipocytes and macrophages that could significantly contribute to inflammation and CVD [22]. Resistin has been demonstrated to increase LDL levels and contribute to insulin resistance $[23,24]$. It also upregulates expression of an inflammatory component called MCP-1 [25]. MCP-1 is produced by multiple cell types, including fibroblasts, monocytes, mesangial and also endothelial cells either constitutively or after induction by oxidative stress [26]. Resistin recruits monocytes and T cells to the sites of inflammation or damaged tissues [27]. Migrated monocytes differentiate into the foam cells in sub endothelial space where they 
initiate to produce fatty streak formations that result in atherosclerotic plaque formation [28]. Some other monocytes differentiate into tissue-resident macrophages with the ability to produce proinflammatory cytokines TNF-a, IL-1, and IL-6 that affect the liver, heart, bones, kidney, and many other tissues $[29,30]$. Of these, the liver releases acute phase proteins like CRP in response to mild inflammation. CRP is believed to cause inflammation on coronary vessels, subsequently leading to myocardial ischemia [31]. On the other hand, long-term elevation of insulin level during obesity, prolongs obesity, creating a positive feedback loop and inflammation changes into the chronic form. Insufficient reperfusion further exacerbates the inflammation in injured myocardium and causes myocardial necrosis [32].

\section{Conclusions}

Our findings support the hypothesis of multiple determinants in the prediction of CVD risk factors in obese adult females. The utility of PCA helped to statistically analyze a comprehensive panel of adipokines and identifying potential candidates for more detailed studies in the future. Based on the results of the PCA, the panel of CRP, MCP-1, and insulin were shown responsible for the most significant variability in cardiovascular disease risk factors, however, further investigations are required to confirm whether our adipokines patterns are associated with the clinical and biological.

\section{Limitations:}

To the best of our knowledge, this was the first study to investigate the adipokines pattern and CVD, however there were a number of limitations. The study was limited to the number of participants and same-sex samples. We only recruited obese females, therefore our results may not be applicable to the general population. Furthermore, we had a small number of predominantly inflammatory adipokines and further studies should look at introducing more adipokines another important limitation is the crosssectional design of the study that prevented us from inferring causality.

\section{Abbreviations}

CVDs: Cardiovascular diseases

TNFa: Tumor necrosis factor-alpha

IL-6: Interleukin-6

IL-1 $\beta$ : Interleukin-1- beta

CRP: C-reactive protein

PAl-1: Plasminogen activator inhibitor-1

MCP-1: Monocyte chemoattractant protein-1 
BMI: Body mass index

RMR: Resting metabolic rate

FBS: Fasting blood surge

Cl: Confidence interval

ANOVA: Analysis of variance

SBP: systolic blood pressure

DBP: Diastolic blood pressure

BFM: Body fat mass

ODP: Obesity degree percentage

WC: Waist circumference

WHR: Waist-hip ratio

FFM: Fat-free mass

SLM: Soft lean mass

\section{Declarations}

Ethics approval(IR.TUMS.VCR.REC.1395.1597) in Tehran University of Medical Science

Consent to publish: Not applicable

Consent for publication: This is formally to submit the article entitled "Principal Component Analysis of Adipocytokines and Insulin Associate with Risk Factors of Cardiovascular Diseases "prepared by the Tehran University of Medical Sciences for review and, hopefully, publication in your prestigious journal. The authors would like to advise that all authors listed have contributed to the work. All authors have agreed to submit the manuscript to BMC Research Notes. No part of the work has been published before.

Availability of data and materials

No funding

Conflict of Interest: None

All of authors declared that they have no competing interests 
Author Contribution: "SP, and SFS designed research; NB conducted research; KhM and HY analyzed data; HY wrote the paper; KhM had primary responsibility for final content. MA helped us in the process of revises and LS and CR improve grammar. All authors read and approved the final manuscript."

\section{Acknowledgment}

The authors thank the directors of the school of Nutritional and Dietetics at Tehran University of medical sciences for allowing them to conduct a comparative cross-sectional study for the purpose of evaluating CVDs risk factors. This study was supported by grants (ID: 95-03-161-33142 and 96-01-161-34479) from Tehran University of Medical Sciences.

There is no conflict of interest in this paper.

\section{References}

1. Braunwald E: Heart disease. 1988.

2. Cosselman KE, Navas-Acien A, Kaufman JDJNRC: Environmental factors in cardiovascular disease. 2015, 12:627.

3. Poirier P, Eckel RHJCar: Obesity and cardiovascular disease. 2002, 4:448-453.

4. Mattu HS, Randeva HSJJoe: Role of adipokines in cardiovascular. 2013, 216:T17-T36.

5. Tesauro M, Canale MP, Rodia G, Di Daniele N, Lauro D, Scuteri A, Cardillo CJCR, Practice: Metabolic syndrome, chronic kidney, and cardiovascular diseases: role of adipokines. 2011, 2011.

6. Smith Jr SCJTAjom: Multiple risk factors for cardiovascular disease and diabetes mellitus. 2007, 120:S3-S11.

7. Das UJN: Is obesity an inflammatory condition? 2001, 17:953-966.

8. Rifai N, Ridker PMJCoil: Inflammatory markers and coronary heart disease. 2002, 13:383-389.

9. Cozlea D, Farcas D, Nagy A, Keresztesi A, Tifrea R, Cozlea L, Carașca EJChsj: The impact of C reactive protein on global cardiovascular risk on patients with coronary artery disease. 2013, 39:225.

10. Flegal KM, Carroll MD, Ogden CL, Curtin LRJJ: Prevalence and trends in obesity among US adults, 1999-2008. 2010, 303:235-241.

11. Ginsberg HN, MacCallum PRJJotcs: The obesity, metabolic syndrome, and type 2 diabetes mellitus pandemic: Part I. Increased cardiovascular disease risk and the importance of atherogenic dyslipidemia in persons with the metabolic syndrome and type 2 diabetes mellitus. 2009, 4:113-119.

12. Després J-P, Lemieux I, Prud'Homme DJB: Treatment of obesity: need to focus on high risk abdominally obese patients. 2001, 322:716-720.

13. Nakamura K, Fuster JJ, Walsh KJJoc: Adipokines: a link between obesity and cardiovascular disease. 2014, 63:250-259.

14. Goldstein BJ, Scalia RJTJoCE, Metabolism: Adiponectin: a novel adipokine linking adipocytes and vascular function. 2004, 89:2563-2568. 
15. Wattanapitayakul SK, Bauer JAJP, therapeutics: Oxidative pathways in cardiovascular disease: roles, mechanisms, and therapeutic implications. 2001, 89:187-206.

16. Jain S, Sharma S, Mandal T, Saxena MJP: Source apportionment of PM10 in Delhi, India using PCAVAPCS, UNMIX and PMF. 2018, 37:107-118.

17. Flehmig G, Scholz M, Klöting N, Fasshauer M, Tönjes A, Stumvoll M, Youn B-S, Blüher MJPo: Identification of adipokine clusters related to parameters of fat mass, insulin sensitivity and inflammation. 2014, 9:e99785.

18. Coppack SWJPotNS: Pro-inflammatory cytokines and adipose tissue. 2001, 60:349-356.

19. Clément K, Langin DJJoim: Regulation of inflammation-related genes in human adipose tissue. 2007, 262:422-430.

20. Slocum C, Kramer C, Genco CJ Joim: Immune dysregulation mediated by the oral microbiome: potential link to chronic inflammation and atherosclerosis. 2016, 280:114-128.

21. Kim C, Park H, Kawada T, Kim J, Lim D, Hubbard N, Kwon B, Erickson KL, Yu RJljoo: Circulating levels of MCP-1 and IL-8 are elevated in human obese subjects and associated with obesity-related parameters. 2006, 30:1347.

22. Wang H, Chu WS, Hemphill C, Elbein SCJTJoCE, Metabolism: Human resistin gene: molecular scanning and evaluation of association with insulin sensitivity and type 2 diabetes in Caucasians. 2002, 87:2520-2524.

23. De Luis D, Sagrado MG, Conde R, Aller R, Izaola OJNh: Resistin levels and inflammatory markers in patients with morbid obesity. 2010, 25:630-634.

24. Park HK, Kwak MK, Kim HJ, Ahima RSJTKjoim: Linking resistin, inflammation, and cardiometabolic diseases. 2017, 32:239.

25. Bokarewa M, Nagaev I, Dahlberg L, Smith U, Tarkowski AJTJol: Resistin, an adipokine with potent proinflammatory properties. 2005, 174:5789-5795.

26. Deshmane SL, Kremlev S, Amini S, Sawaya BEJJoi, research c: Monocyte chemoattractant protein-1 (MCP-1): an overview. 2009, 29:313-326.

27. Carr MW, Roth SJ, Luther E, Rose SS, Springer TAJPotNAoS: Monocyte chemoattractant protein 1 acts as a T-lymphocyte chemoattractant. 1994, 91:3652-3656.

28. Bobryshev YVJM: Monocyte recruitment and foam cell formation in atherosclerosis. 2006, 37:208222.

29. Silswal N, Singh AK, Aruna B, Mukhopadhyay S, Ghosh S, Ehtesham NZJB, communications br: Human resistin stimulates the pro-inflammatory cytokines TNF- $a$ and IL-12 in macrophages by NFKB-dependent pathway. 2005, 334:1092-1101.

30. Lehrke M, Reilly MP, Millington SC, Iqbal N, Rader DJ, Lazar MAJPm: An inflammatory cascade leading to hyperresistinemia in humans. 2004, 1:e45.

31. Heinrich J, Schulte H, Schönfeld R, Köhler E, Assmann GJT, haemostasis: Association of variables of coagulation, fibrinolysis and acute-phase with atherosclerosis in coronary and peripheral arteries and 
those arteries supplying the brain. 1995, 73:374-379.

32. Pietilä K, Harmoinen A, Hermens W, Simoons M, Van de Werf F, Verstraete MJEhj: Serum C-reactive protein and infarct size in myocardial infarct patients with a closed versus an open infarct-related coronary artery after thrombolytic therapy. 1993, 14:915-919.

\section{Tables}

Table 1. Characteristics of the study participants

\begin{tabular}{|c|c|c|c|c|}
\hline Variable & Mean & SD & Minimum & Maximum \\
\hline Age (years) & 36.52 & 8.32 & 18.00 & 50.00 \\
\hline Body weight (kg) & 78.75 & 11.51 & 1.00 & 119.50 \\
\hline BMI (kg/m $\left.{ }^{2}\right)$ & 30.33 & 3.65 & 25.00 & 40.70 \\
\hline RMR and blood pressure parameters \\
\hline RMR (kcal/d) & 1566.51 & 253.49 & 952.0 & 2467.00 \\
\hline SBP (mmHg) & 113.11 & 14.05 & 76.00 & 173.00 \\
\hline DBP (mmHg) & 78.13 & 9.36 & 51.00 & 111.00 \\
\hline \multicolumn{5}{|c|}{ Blood parameters } \\
\hline Serum Cholesterol (g/dl) & 185.61 & 38.48 & 104.00 & 433.00 \\
\hline \multicolumn{6}{|c|}{ TG (g/dl) } & 118.39 & 64.54 & 37.00 & 512.00 \\
\hline LDL (mg/dl) & 96.98 & 26.86 & 34.00 & 282.00 \\
\hline HDL (mg/dl) & 47.77 & 10.87 & 18.00 & 84.00 \\
\hline Hs.CRP (mg/dl) & 4.05 & 4.54 & 0.00 & 22.73 \\
\hline FBS (mmol) & 4.92 & 0.63 & 3.72 & 11.22 \\
\hline HOMA & 3.33 & 1.52 & .39 & 16.59 \\
\hline \multicolumn{7}{|c|}{ Body composition analysis } \\
\hline BFM (Kg) & 33.43 & 7.60 & 19.40 & 53.20 \\
\hline FFM (Kg) & 46.62 & 5.43 & 35.30 & 67.70 \\
\hline SLM (Kg) & 43.84 & 5.16 & 26.10 & 63.80 \\
\hline SMM (Kg) & 25.59 & 3.22 & 18.90 & 37.90 \\
\hline ODP & 142.86 & 17.33 & 116.00 & 189.00 \\
\hline WC (cm) & 98.45 & 9.24 & 80.10 & 123.20 \\
\hline FFMI & 18.32 & 7.78 & 14.60 & 147.80 \\
\hline FMI & 12.91 & 2.96 & 6.90 & 21.20 \\
\hline WHR & 0.93 & 0.05 & 0.81 & 1.08 \\
\hline
\end{tabular}

SD: Standard deviation; BFM: Body fat mass; SLM: Soft lean mass; ODP: Obesity degree percentage; BMI: Body mass index; TG: Triglyceride; LDL: Low density lipoprotein; HDL; High density lipoprotein; FFM: fat free mass; RMR, resting metabolic rate; SBP: systolic blood pressure; DBP: diastolic blood pressure; HOMA: homeostasis model insulin resistance index; hs-CRP: high sensitivity C-reactive protein; WC: Waist Circumference; WHR: Waist hip ratio; FFMI: fat free mass index; FMI: fat mass index. 
Table 2: Association between demographics, medical and biomedical characteristics with patterns 1 of Adipokines.

\begin{tabular}{|c|c|c|c|c|}
\hline \multirow[t]{2}{*}{ Variable } & \multicolumn{4}{|c|}{ Pattern 1 group } \\
\hline & $\mathrm{B}$ & 95.0\% Confidence Interval & & \\
\hline Age (years) & -0.12 & -0.81 to 0.56 & 0.71 & 0.71 \\
\hline Weight (kg) & -0.92 & -1.87 to 0.02 & 0.05 & 0.05 \\
\hline BMI $\left(\mathrm{kg} / \mathrm{m}^{2}\right)$ & -0.34 & -0.63 to -0.04 & 0.02 & 0.06 \\
\hline \multicolumn{5}{|c|}{ RMR and blood pressure parameters } \\
\hline RMR (kcal/d) & -15.58 & -36.94 to 5.78 & 0.15 & 0.13 \\
\hline SBP (mmHg) & -0.89 & -2.08 to 0.28 & 0.13 & 0.11 \\
\hline $\mathrm{DBP}(\mathrm{mmHg})$ & -0.93 & -1.72 to -0.14 & 0.02 & 0.06 \\
\hline \multicolumn{5}{|c|}{ Blood parameters } \\
\hline Cholesterol (g/dl) & -0.26 & -5.42 to 0.89 & 0.15 & 0.13 \\
\hline TG $(\mathrm{g} / \mathrm{dl})$ & 0.11 & -5.20 to 5.43 & 0.96 & 0.94 \\
\hline $\mathrm{LDL}(\mathrm{mg} / \mathrm{dl})$ & -1.30 & -1.35 to 0.90 & 0.24 & 0.17 \\
\hline $\mathrm{HDL}(\mathrm{mg} / \mathrm{dl})$ & -0.60 & -1.49 to 0.28 & 0.18 & 0.14 \\
\hline hs.CRP (mg/dl) & 0.10 & -0.28 to 0.46 & 0.57 & 0.60 \\
\hline HOMA & 0.01 & -0.11 to 0.14 & 0.83 & 0.81 \\
\hline FBS (mmol) & 0.03 & -0.02 to 0.08 & 0.23 & 0.15 \\
\hline \multicolumn{5}{|c|}{ Body composition parameters } \\
\hline BFM (kg) & -0.98 & -1.60 to -0.37 & 0.002 & 0.001 \\
\hline FFM (kg) & -0.03 & -0.48 to 0.41 & 0.86 & 0.85 \\
\hline SLM (kg) & -0.00 & -0.43 to 0.42 & 0.98 & 0.97 \\
\hline SMM (kg) & -0.01 & -0.27 to 0.25 & 0.91 & 0.93 \\
\hline ODP & -1.57 & -2.98 to -0.15 & 0.03 & 0.02 \\
\hline WC (cm) & -1.07 & -1.82 to -0.32 & 0.005 & 0.005 \\
\hline FFMI & -0.13 & -0.77 to -0.50 & 0.68 & 0.67 \\
\hline FMI & -0.37 & -0.61 to -0.13 & 0.003 & 0.002 \\
\hline WHR & -0.00 & -0.01 to -0.00 & 0.004 & 0.004 \\
\hline
\end{tabular}

$M e a n \pm S D:$ mean \pm standard deviation; $P$-value_ $\alpha$ : $p$-value result from $A N O V A ; P$-value_ $\beta$ P: $P$-value result from Generalized Linear mode(General linear model for adjusting age, gender, and total energy intake)l; BFM: Body fat mass; SLM: Soft lean mass; ODP: Obesity degree percentage; BMI: Body mass index; TG: Triglyceride; LDL: Low density lipoprotein; HDL; High density lipoprotein; FFM: fat free mass; RMR, resting metabolic rate; SBP: systolic blood pressure; DBP: diastolic blood pressure; HOMA: homeostasis model insulin resistance index; hs-CRP: high sensitivity C-reactive protein; WC: Waist Circumference; WHR: Waist hip ratio; FFMI: fat free mass index; FMI: fat mass index.

Table 3: Association between demographics, medical and biomedical characteristics with patterns 2 of Adipokines. 


\begin{tabular}{|c|c|c|c|c|}
\hline \multirow[t]{2}{*}{ Variable } & \multicolumn{4}{|c|}{ Pattern 2 group } \\
\hline & $\mathrm{B}$ & 95.0\% Confidence Interval & & \\
\hline Age (years) & -0.88 & -1.57 to -0.20 & 0.010 & 0.012 \\
\hline Weight (kg) & 1.57 & 0.59 to 2.47 & 0.001 & 0.003 \\
\hline BMI $\left(\mathrm{kg} / \mathrm{m}^{2}\right)$ & 0.48 & 0.18 to 0.77 & 0.002 & 0002 \\
\hline \multicolumn{5}{|c|}{ RMR and blood pressure parameters } \\
\hline RMR (kcal/d) & 42.12 & 21.62 to 62.63 & 0.000 & 0.000 \\
\hline $\mathrm{SBP}(\mathrm{mmHg})$ & 0.67 & -0.49 to 1.87 & 0.25 & 0.21 \\
\hline $\mathrm{DBP}(\mathrm{mmHg})$ & 1.88 & 0.60 to 2.14 & 0.00 & 0.001 \\
\hline \multicolumn{5}{|c|}{ Blood parameters } \\
\hline Cholesterol (g/dl) & 0.93 & -2.24 to 4.12 & 0.56 & 0.52 \\
\hline TG $(\mathrm{g} / \mathrm{dl})$ & 8.44 & 3.18 to 13.70 & 0.002 & 0.002 \\
\hline $\mathrm{LDL}(\mathrm{mg} / \mathrm{dl})$ & 1.28 & -0.93 to 3.50 & 0.25 & 0.17 \\
\hline HDL (mg/dl) & -0.164 & -2.53 to -0.76 & 0.000 & 0.000 \\
\hline hs.CRP (mg/dl) & 2.11 & 1.82 to 2.41 & 0.000 & 0.000 \\
\hline HOMA & 0.58 & 0.48 to 0.69 & 0.000 & 0.000 \\
\hline FBS (mmol) & 0.02 & -0.02 to 0.07 & 0.30 & 0.36 \\
\hline \multicolumn{5}{|c|}{ Body composition analysis } \\
\hline BFM (kg) & 0.78 & 0.16 to 1.40 & 0.013 & 0.012 \\
\hline FFM (kg) & 0.61 & 0.16 to 1.05 & 0.007 & 0.009 \\
\hline SLM (kg) & 0.62 & 0.20 to 1.04 & 0.004 & 0.005 \\
\hline SMM (kg) & 0.38 & 0.12 to 0.65 & 0.004 & 0.005 \\
\hline ODP & 2.23 & 0.82 to 3.64 & 0.002 & 0.002 \\
\hline $\mathrm{WC}(\mathrm{cm})$ & 1.15 & 0.40 to 1.90 & 0.003 & 0.003 \\
\hline FFMI & -0.08 & -0.73 to 0.55 & 0.78 & 0.77 \\
\hline FMI & 0.26 & 0.02 to 0.51 & 0.03 & 0.026 \\
\hline WHR & 0.004 & 0.000 to 0.008 & 0.084 & 0.078 \\
\hline
\end{tabular}

$M e a n \pm S D$ : mean \pm standard deviation; $P$-value_ $\alpha$ : $p$-value result from ANOVA; $P$-value _ $\beta$ P: P-value result from Generalized Linear mode(General linear model for adjusting age, gender, and total energy intake)l; BFM: Body fat mass; SLM: Soft lean mass; ODP: Obesity degree percentage; BMI: Body mass index; TG: Triglyceride; LDL: Low density lipoprotein; HDL; High density lipoprotein; FFM: fat free mass; RMR, resting metabolic rate; SBP: systolic blood pressure; DBP: diastolic blood pressure; HOMA: homeostasis model insulin resistance index; hs-CRP: high sensitivity C-reactive protein; WC: Waist Circumference; WHR: Waist hip ratio; FFMI: fat free mass index; FMI: fat mass index.

\section{Supplementary Files}

This is a list of supplementary files associated with this preprint. Click to download.

- tableS3.docx 
- tableS2.docx

- tableS1.docx 